\title{
Amoebic Liver Abscess
}

\section{A disease native to Oman?}

Nenad Pandak, ${ }^{1}$ "Sirous Golchinheydari, ${ }^{1}$ Asmaa S. Mahdi, ${ }^{1}$ Ali Al Majrafi, ${ }^{2}$ Stephen S. Deenadayalan, ${ }^{3}$ Faryal Khamis ${ }^{1}$

\begin{abstract}
Objectives: Amoebic liver abscess (ALA) is endemic to many areas of the world. This study sought to investigate the epidemiology, presentation, laboratory tests and imaging characteristics of ALA in Oman and ultimately determine whether it is native to Oman or originated abroad. Methods: This case series study was conducted at the Royal Hospital, Muscat, Oman, from January 2013 to December 2017 with patients older than 13 years and having a discharge diagnosis of ALA. Patient data were extracted from the Royal Hospital patient database. Results: 22 patients were included in the study-18 Omani patients and four expatriates. Only two Omanis had a history of traveling abroad. There were 15 male patients and seven were female with an average age of 45.2 years. The most common presentation was abdominal pain, which was seen in 17 patients. Fever was seen in 13 patients. Alanine transferase was found to be elevated in 13 patients. The majority of patients (90\%) had no symptomatic infections prior to developing ALA. Conclusion: The data suggests that ALA is endemic to Oman, considering the high number of local patients and lack of travel abroad in this population. As the number of patients treated for ALA is rather small, it can be concluded that the occurrence of ALA is much lower in Oman than in other endemic areas. The majority of patients had no prior symptomatic infections; thus, a method of control involves screening to prevent amoebic spread.
\end{abstract}

Keywords: Amoebic Liver Abscess; Amoebiasis; Liver Abscess; Entamoeba histolytica; Oman.

\section{Advances in KNOWLedge}

Amoebic liver abscess is endemic to Oman.

A large proportion of patients are asymptomatic carriers in Oman.

This study's results are contrary to what other countries in the region with a similar migrant population have reported.

\section{Application in Patient Care}

Healthcare professionals should actively look for and treat infected patients to limit the amoebic spread.

A MOEBIC LIVER ABSCESS (ALA) IS A SECONDARY result of amoebiasis caused by Entamoeba histolytica, a protozoan transmitted mainly through the faecal-oral route. This can occur through the ingestion of water or food that is contaminated with cysts excreted via faeces. ${ }^{1}$ Amoebic infections are mostly asymptomatic, but invasive intestinal disease can occur in the colon when the invasive trophozoite form invades colonic mucosa and activates a strong immune response, thus causing further tissue damage. ${ }^{2}$ Access to mesenterial vessels can result in haematogenous amoebic dissemination to extra-intestinal sites, potentially causing a liver abscess. $^{3}$ ALA is the most common extra-intestinal manifestation and has a widespread global impact; it primarily affects developing countries with poor hygiene and inadequate sanitation. ${ }^{4}$ High rates of ALA are seen in the Indian subcontinent, East Asia, Africa and Central and South America. ${ }^{5}$ Immigration is an important factor for cases discovered in non-endemic areas. Despite the above, ALA reports from countries in the Arabian Peninsula are scarce. Studies in Saudi Arabia and Qatar show that a very large proportion of ALA patients are either foreigners or local citizens that have travelled to endemic areas. ${ }^{6,7}$ To the best of the authors' knowledge, the epidemiological situation related to ALA in Oman remains unknown. Similar to other Gulf countries, Oman has a large migrant worker community from endemic areas in Asia and as of 2020, they account for approximately $40 \%$ of the population. ${ }^{8}$ It is, therefore, natural to assume that the majority of ALA patients would also be migrants. Accordingly, this retrospective study aimed to establish the epidemiological and clinical characteristics of ALA cases in Oman and determine if Oman is a region endemic to ALA or if most of the cases originate elsewhere.

\section{Methods}

This study was carried out at the Royal Hospital in Muscat, Oman and data was extracted from the hospital database. Patients older than 13 years of age who were diagnosed with ALA and admitted to the Royal Hospital between January 2013 and December 2017 were included. The diagnosis was confirmed via 
the commercial immunoglobulin G (IgG) anti-amoebic assay (R-BiopharmAG, Darmstadt, Germany). The extracted data included patient biodata and history, comorbidities, laboratory values, radiological imaging reports and procedures carried out. These were analysed using descriptive statistical methods with Microsoft Office Word, Version 2017 (Microsoft Corp., Redmond, Washington, USA). Ethical approval was obtained from The Royal Hospital Scientific Research Committee (code: SRC\#32/2018).

\section{Results}

During the five-year period considered for this study, 75 patients were treated for liver abscess. Of these, 53 were confirmed to have pyogenic liver abscess and 22 patients were confirmed and treated for ALA. Eighteen patients were Omanis and four were expatriates; of the latter, three were from India and one was from Pakistan. There were 15 (68\%) male patients and seven (32\%) were female. The patient ages ranged from 16 to 77 years and the average age was 45.2 . The average age of the men was higher (47.4) than the women (40.6).

The most common presentation, as seen in $17(77 \%)$ patients, was abdominal pain. Fever was a common symptom in 13 patients (59\%), while jaundice was far less common and presented only in three (14\%) patients. Diabetes mellitus was the most common comorbidity found in patients with ALA. A total of seven patients had comorbidities, out of which five (71\%) had diabetes mellitus while the rest had hepatobiliary disease. All but one patient was admitted to the general ward, while that one patient was admitted to the ICU. None of the patients required urgent surgery and none presented with sepsis or septic shock.

Laboratory testing was done for alanine aminotransferase (ALT), bilirubin, albumin, leukocyte and platelet levels. A significant number of patients ( $\mathrm{n}=$ 13; 59\%) presented with above-normal ALT, while nine (41\%) patients presented with normal ALT levels. However, bilirubin did not follow the same trend. The majority of patients $(n=13 ; 59 \%)$ had normal bilirubin levels, while nine (41\%) had raised bilirubin levels. Albumin levels were similar to bilirubin; it was normal in the majority of patients $(n=20 ; 91 \%)$ and only two (9\%) had abnormal albumin levels. As expected, most patients presented with leukocytosis. Exactly half (50\%) the patients had leukocyte counts in the range of $10-20 / \mu \mathrm{L}$ of blood, while seven (32\%) patients had counts of more than $20 / \mu \mathrm{L}$ of blood. Only one (4\%) patient had an abnormal platelet count.
The anatomical position of the abscess was established for each patient using multiple radiological imaging techniques. A total of 19 (86\%) patients were found to have an abscess in the right lobe, while three (14\%) patients had bilateral abscesses. The presentation of a single abscess was found in 15 (68\%) patients, while multiple abscesses were found in seven (32\%). Percutaneous drainage was done for 18 patients (82\%).

No prior infections were found in $90 \%$ of patients. The remaining 10\% had previously had gastrointestinal infections. This shows that the majority were asymptomatic carriers.

The outcome of all the patients was good. None of the patients died and they were all cured and discharged from the hospital.

\section{Discussion}

The available data show that a significant number of liver abscesses are reported to be pyogenic in origin and not amoebic. ${ }^{9}$ Invasive amoebiasis, such as ALA, is attributed to 50 million infections worldwide. ${ }^{2}$ The incidence rate in the US is $8.5 / 100,000$ hospital admissions, with extremely low mortality. ${ }^{10}$ The known endemic area of Sri Lanka has reported an incidence of 3-9/10,000 hospital admissions. ${ }^{11}$ Taiwan, another known endemic area, has attributed the sharp drop in the high prevalence rate of ALA to improved sanitation. ${ }^{12,13}$ At the same time, the mortality rate was reported to be $2.4 \%$. To the best of the authors' knowledge, there is no data about the prevalence of ALA in Oman and its neighbouring countries in the Arabian Peninsula. It can be assumed that ALA occurrence in Oman is low and, as shown by this study, the mortality rate is considerably lower when compared to the regions indicated in other studies. Notably, the present study was conducted at a single centre, the Royal Hospital in Muscat, and does not represent the incidence of ALA in Oman. However, the Royal Hospital is a tertiary care centre and the biggest hospital in the country, containing the largest infectious diseases department in the country.

Immunocompromised hosts are prone to developing ALA. ${ }^{14}$ It is well known that diabetes mellitus is the most common predisposing factor to pyogenic liver abscess, but no such conclusion seems to have been drawn about ALA. ${ }^{6,7}$ The data from the present study suggest that diabetes mellitus is also a major risk factor for ALA, as approximately $71 \%$ of the patients with comorbidities had diabetes mellitus. It is also well known that the ALA is more common in men and, as one study from India showed, that the gender 
proportion of men can rise up to $93 \%{ }^{15}$ Similarly, a report from Qatar showed that only $9 \%$ of their ALA patients were female. ${ }^{7}$ In the present study, about twothirds of the patients were male, but this proportion could be biased due to the cohort size. It has previously been reported that ALA most often occurs in the age group of $18-50$ years and the present study's data is in agreement with this. ${ }^{16}$ In the US, most of the ALA cases are found among the immigrant population, mostly from Mexico. ${ }^{17,18}$ Reports from Qatar and Saudi Arabia show that the majority of patients are either expatriates or had a history of travel to endemic areas. ${ }^{6,7}$ In contrast, most of the patients in the present study were locals and only two of them had a history of travel. One travelled to Pakistan three months prior to his illness and the other one visited India a year before the onset of his illness. This indicates that, unlike Saudi Arabia and Qatar, ALA is indigenous to Oman.

Coinciding with the study data, abdominal pain in the right upper quadrant and fever have been reported to be the most common forms of presentation. ${ }^{17}$ Septic shock or admission to the ICU, which can be used as a parameter of disease severity, does not seem to be common among ALA patients as this is not addressed in the existing literature. The present study's results were similar, although one of the 22 patients was admitted and treated in the ICU.

The majority of the patients (86\%) in the present study had abscesses in the right liver lobe and the left lobe was involved only in patients with bilateral abscesses. Other studies have showed that about half of ALA cases involve the right lobe and the rest are left-sided or bilateral. ${ }^{9}$ The right lobe of the liver has more blood supply, which may explains why more lesions are found on the right side of the liver. It has been reported that a rather small number of patients have bilateral lesions; this aligns with the present study's data, which shows that $14 \%$ of the patients had bilateral lesions. ${ }^{13}$ Previously published studies state that ALA cases usually involve single abscesses, as was found in the present study's cohort.

Amoebiasis occurs after the ingestion of E. hystolitica cysts found in faecally contaminated water or food. The cyst migrates to the colon and undergoes excystation, releasing trophozoites that can penetrate colonic mucosa and the hepatic portal circulation, resulting in haematogenous spread. This spread often leads to ALA formation, but in rare cases, the trophozoite can even spread to other organs. ${ }^{2}$ Almost $90 \%$ of infected patients are asymptomatic and fewer than $1 \%$ of patients with amoebic colitis have associated extra-intestinal disease manifestation, most often ALA. ${ }^{11}$ This explains why only $10 \%$ of the study patients previously had a history of intestinal infection and it also indicates that, prior to the ALA, these asymptomatic patients represented a significant risk to their environment.

It is estimated that E. hystolitica globally affects around 50 million people every year, causing more than 10,000 deaths in $2013 .^{2}$ This includes occurrences of intestinal and extra-intestinal amoebiasis. Mortality varies around the world and depends on whether the country is in an endemic area as well as the quality of and access to healthcare. There is no data regarding the ALA mortality rate of countries in the Arabian Peninsula. Notably, there were no fatal outcomes connected to ALA treatment in the present study, most probably because of easy access to high-quality healthcare facilities in Oman.

The main limitation of this study is its singlecentre design and rather small sample size. Due to this, it is not representative of the ALA epidemiology in Oman, but it provides good insights into the epidemiological situation in the country and serves as a good starting point for further studies.

\section{Conclusion}

As the majority of the study patients were Omanis without any history of travel to ALA endemic areas, it can be concluded with high certainty that Oman is an endemic area but with considerably lower ALA occurrence than other endemic areas close by. In addition, the majority of patients were asymptomatic cyst passers prior to their diagnosis and represented a significant risk for others; therefore, it is important to actively look for infected persons and begin early treatment to prevent the amoebic spread.

\section{CONFLICT OF INTEREST}

The authors of this article declare that there is no conflict of interest.

\section{FUNDING}

No funding was received for this study.

\section{AUTHORS' CONTRIBUTION}

NP and SG designed the research. Data collection was carried out by all authors. SG analysed the results and drafted the manuscript. All authors contributed with suggestions and amendments to the initial version of the manuscript. All authors approved the final version of the manuscript. 


\section{References}

1. Kantor M, Abrantes A, Estevez A, Schiller A, Torrent J, Gascon J, et al. Entamoeba histolytica: Updates in clinical manifestation, pathogenesis, and vaccine development. Can J Gastroenterol Hepatol 2018; 2018:1-6. https://doi.org/10.1155/2018/4601420.

2. Tharmaratnam T, Kumanan T, Iskandar M, D'Urzo K, GopeeRamanan P, Loganathan M, et al. Entamoeba histolytica and amoebic liver abscess in northern Sri Lanka: A public health problem. Trop Med Health 2020; 48:1-13. https://doi.org/10.1 186/s41182-020-0193-2.

3. Drinić M, Raninger A, Zraunig A, Astelbauer F, Leitsch D, Obwaller A. Activity of methylgerambullin from Glycosmis species (Rutaceae) against Entamoeba histolytica and Giardia duodenalis in vitro. Int J Parasitol Drugs Drug Resist 2019; 10:109-17. https://doi.org/10.1016/j.ijpddr.2019.08.001.

4. Torre A, Kershenobich D. Amebic liver abscess. Ann Hepatol 2002; 1:45-7. https://doi.org/10.1016/S1665-2681(19)32192-1.

5. Chou A, Austin RL. Entamoeba histolytica. Treasure Island, USA: StatPearls Publishing, 2020.

6. Albenmousa A, Sanai FM, Singhal A, Babatin MA, AlZanbagiAA, Al-Otaibi MM, et al. Liver abscess presentation and management in Saudi Arabia and the United Kingdom. Ann Saudi Med 2011; 31:528-32. https://doi.org/10.4103/0256-4947.84635.

7. Abbas M, Khan F, Muhsin S, Al-Dehwe B, Abukamar M, Elzouki A. Epidemiology, clinical features and outcome of liver abscess: A single reference center experience in Qatar. Oman Med J 2014; 29:260-3. https://doi.org/10.5001/omj.2014.69.

8. National Centre For Statistics and Information. From: https:// www.ncsi.gov.om/Pages/NCSI.aspx Accessed: Mar 2021

9. Akhondi H, Sabih DE. Liver abscess. Treasure Island, USA: StatPearls Publishing, 2020.
10. Seeto RK, Rockey DC. Amebic liver abscess: epidemiology, clinical features, and outcome. West J Med 1999; 170:104-9.

11. KannathasanS,MurugananthanA,KumananT,deSilvaN,Rajeshkannan N, Haque R, et al. Epidemiology and factors associated with amoebic liver abscess in northern Sri Lanka. BMC Public Health 2018; 18:1-8. https://doi.org/10.1186/s12889-018-5036-2.

12. Chuah SK, Chang-Chien CS, Sheen IS, Lin HH, Chiou SS, Chiu CT, et al. The prognostic factors of severe amebic liver abscess: A retrospective study of 125 cases. Am J Trop Med Hyg 1992; 46:398-402. https://doi.org/10.4269/ajtmh.1992.46.398.

13. Chen H, Lin I, Wu C, Lee Y, Bair M. Clinical manifestations and risk factors of amebic liver abscess in Southeast Taiwan compared with other regions of Taiwan. Am J Trop Med Hyg 2013; 89:1214-18. https://doi.org/10.4269/ajtmh.13-0300.

14. Sayek I, Onat D. Pyogenic and amebic liver abscess. In: Holzheimer RG, Mannick JA, Eds. Surgical Treatment: Evidence-Based and Problem-Oriented. Munich, Germany: Zuckschwerdt, 2001.

15. Singh A, Banerjee T, Kumar R, Shukla S. Prevalence of cases of amebic liver abscess in a tertiary care centre in India: A study on risk factors, associated microflora and strain variation of Entamoeba histolytica. PLoS One 2019; 14:e0214880. https:// doi.org/10.1371/journal.pone.0214880.

16. Prakash V, Jackson-Akers JY, Oliver TI. Amebic liver abscess. Treasure Island, USA: StatPearls Publishing, 2019. From: https://europepmc.org/article/NBK/nbk430832\#impact Accessed: Mar 2021.

17. Giorgio A, Esposito V, Farella N, Di Sarno A, Liorre G, DE Stefano M, et al. Amebic liver abscesses: A new epidemiological trend in a non-endemic area? In Vivo 2009; 23:1027-30.

18. Hoffner R, Kilaghbian T, Esekogwu V, Henderson S. Common presentations of amebic liver abscess. Ann Emerg Med 1999; 34:351-5. https://doi.org/10.1016/s0196-0644(99)70130-7. 